\title{
ASSESSMENT OF LANDSLIDE-INDUCED DISPLACEMENT AND DEFORMATION OF ABOVE-GROUND OBJECTS USING UAV-BORNE AND AIRBORNE LASER SCANNING DATA
}

\author{
T. Zieher ${ }^{1 *}$, M. Bremer ${ }^{1,2}$, M. Rutzinger ${ }^{1,2}$, J. Pfeiffer ${ }^{1,2}$, P. Fritzmann ${ }^{3}$, V. Wichmann ${ }^{4}$ \\ ${ }^{1}$ Institute for Interdisciplinary Mountain Research, Austrian Academy of Sciences, Technikerstr. 21a, 6020 Innsbruck, Austria \\ (thomas.zieher, jan.pfeiffer, martin.rutzinger)@oeaw.ac.at \\ ${ }^{2}$ Institute for Geography, University of Innsbruck, Innrain 52f, 6020 Innsbruck, Austria \\ magnus.bremer@uibk.ac.at \\ ${ }^{3}$ Federal state of Tyrol, Division of Geoinformation, Herrengasse 3, 6020 Innsbruck, Austria \\ patrick.fritzmann@tirol.gv.at \\ ${ }^{4}$ Laserdata GmbH, Technikerstr. 21a, 6020 Innsbruck, Austria \\ wichmann@laserdata.at
}

Commission II, WG II/10

KEY WORDS: Landslide monitoring, light detection and ranging, unmanned aerial vehicle, RIEGL RiCOPTER, displacement vectors

\begin{abstract}
:
Multi-temporal 3D point clouds acquired with a laser scanner can be efficiently used for an area-wide assessment of landslide-induced surface changes. In the present study, displacements of the Vögelsberg landslide (Tyrol, Austria) are assessed based on available data acquired with airborne laser scanning (ALS) in 2013 and data acquired with an unmanned aerial vehicle (UAV) equipped with a laser scanner (ULS) in 2018. Following the data pre-processing steps including registration and ground filtering, buildings are segmented and extracted from the datasets. The roofs, represented as multi-temporal 3D point clouds are then used to derive displacement vectors with a novel matching tool based on the iterative closest point (ICP) algorithm. The resulting mean annual displacements are compared to the results of a geodetic monitoring based on an automatic tracking total station (ATTS) measuring 53 retroreflective prisms across the study area every hour since May 2016. In general, the results are in agreement concerning the mean annual magnitude (ATTS: $6.4 \mathrm{~cm}$ within 2.2 years, $2.9 \mathrm{~cm} \mathrm{a}^{-1}$; laser scanning data: $13.2 \mathrm{~cm}$ within 5.4 years, $2.4 \mathrm{~cm} \mathrm{a}^{-1}$ ) and direction of the derived displacements. The analysis of the laser scanning data proved suitable for deriving long-term landslide displacements and can provide additional information about the deformation of single roofs.
\end{abstract}

\section{INTRODUCTION}

Multi-temporal laser scanning has become a cost-efficient data acquisition technique for buildings change detection analyses (Wujanz et al., 2018). Laser scanning technology providing buildings high-resolution topographic information became a standard method in landslide research for landslide detection, mapping, and characterisation, hazard assessment and providing information for susceptibility analysis, modelling and monitoring (including surface displacement and volume budget calculations) (Pánek and Klimeš, 2016). Challenges in operational monitoring concepts comprise uncertainties in data quality due to vegetation cover, surface roughness, platform characteristic such as range and incidence angle effects, and registration errors (Jaboyedoff et al., 2012; Telling et al., 2017).

Analyses of gravitational mass movements based on laser scanning data in general rely on either (i) high-resolution digital elevation models (e.g. raster grids and triangulated irregular networks) Eeckhaut et al. (2007); Monserrat and Crosetto (2008); Břežný and Pánek (2017) or (ii) approaches working with 3D point clouds (Gauthier et al., 2018; Mayr et al., 2017; Pfeiffer et al., 2018; Riquelme et al., 2015). The high availability of different sensing and mapping approaches make the combination and integration of information from different sensors and platforms necessary (Mandlburger et al., 2017; Zieher et al., 2018). The

\footnotetext{
${ }^{*}$ Corresponding author: thomas.zieher@oeaw.ac.at
}

advent of unmanned aerial vehicles as platforms for laser scanners (ULS) makes it necessary to investigate how the respective 3D point clouds can be used for analysing landslide displacements by enriching existing ALS point cloud data supporting area-wide monitoring concepts (Lin et al., 2011; Wallace et al., 2012; Wieser et al., 2016).

The present study aims at deriving 3D displacement vectors from extracted roofs represented in multi-temporal 3D point clouds acquired with airborne laser scanning techniques. The results are compared with a geodetic displacement monitoring using an automatic tracking total station (ATTS). The main objectives and aims of the paper are (i) to automatically extract roofs from 3D point clouds, (ii) introduce and apply a novel change detection algorithm for 3D point clouds and (iii) analyse and compare the resulting displacement vectors with daily measurements of an ATTS.

\section{STUDY AREA}

The Vögelsberg landslide is located in the province of Tyrol (Austria), to the south of the city of Wattens in the Watten valley (Fig. 1). Generally, the north-east facing slope ranging from 750 to $2100 \mathrm{~m}$ a.s.1. is characterized by a deep-seated gravitational slope deformation (DGSGD). Recently, the lower part of the slope showed increased displacement rates, endangering houses, infrastructure and arable land. The landslide within this part of 
the slope ranging from 750 to $1000 \mathrm{~m}$ a.s.l. and a lateral extension of approximately $500 \mathrm{~m}$ can be described as a rock slump - debris slide (Cruden and Varnes, 1996). Since May 2016 the slope is being monitored by means of an ATTS featuring hourly measurements of installed reflectors and periodical terrestrial laser scans, both from the opposite side of the valley. Furthermore, a ULS campaign was carried out in August 2018, covering the active part of the landslide.

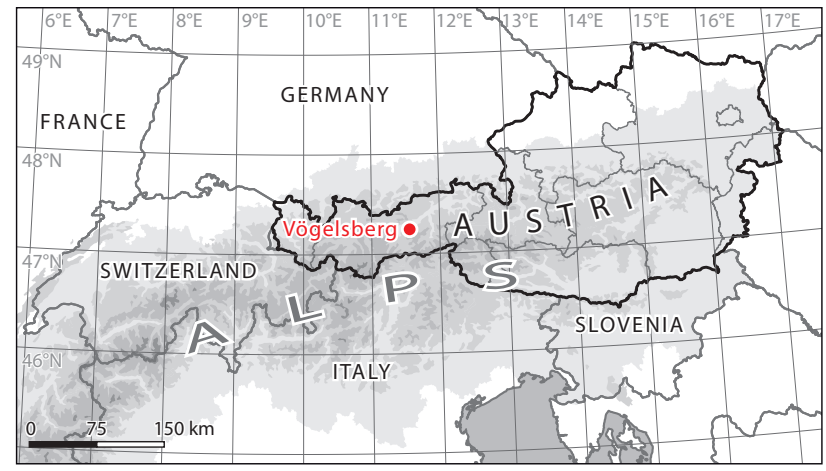

Figure 1. Location of the Vögelsberg landslide in the province of Tyrol, Austria.

\section{MATERIALS AND METHODS}

\subsection{Automatic tracking total station}

With the help of an ATTS hourly measurements of 53 installed retroreflecting prisms throughout the study area were conducted from the opposite side of the valley. Measurement distances range between about 600 to $1700 \mathrm{~m}$. The location of the ATTS is shown in Fig. 2. To derive the displacement vectors based on the daily means of the ATTS, representing the general movement direction and magnitude, the first principal components were computed from the respective coordinates (Fig. 3). The first principal component can be understood as a linear approximation of the 3D data points where the sum of squares of the Euclidian distance is minimized iteratively. With this processing step the displacement vector was fitted to the scattering $3 \mathrm{D}$ data while assuming a linear movement (in space, not in time) of the measured retroreflecting prisms. For the comparison with the results based on the laser scanning data, mean annual vectors were computed.

\subsection{Airborne laser scanning}

Airborne laser scanning (ALS) is a dynamic, active remote sensing technique, with a scanner mounted to an aircraft. In the province of Tyrol (Austria), airborne laser scanning (ALS) data are available area-wide from acquisition campaigns carried out from 2006 to 2009 . On the valley floors the data were updated 2012 to 2013. In the present study the ALS point cloud acquired in 2013 was used. In the respective ALS campaign the data was acquired with a Eurocopter AS350 with a Riegl LMS-Q680i LiDAR instrument on board and a flight altitude of about $600 \mathrm{~m}$. Throughout the covered area a minimum point density of at least 8 points per square metre were demanded. The data acquisition and pre-processing was carried out by the company AVT Consultants for Surveying Engineering. The flight trajectories of the ALS acquisition from 2013 are shown in Fig. 2.

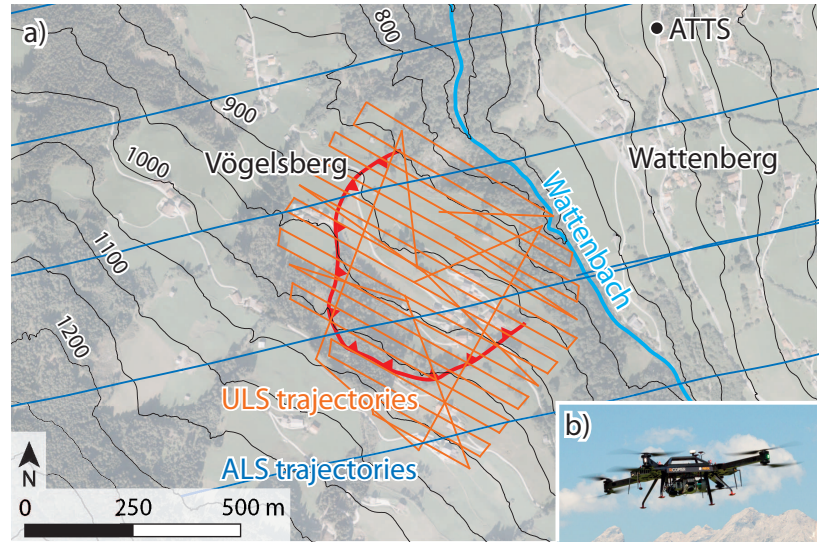

Figure 2. Setup of the landslide monitoring. ULS flight trajectories (orange) and ALS trajectories (blue) over the active part of the Vögelsberg landslide (red) (a) and the RIEGL

RiCOPTER equipped with a VUX-11R laser scanner (b). The location of the automatic tracking total station (ATTS) is shown in (a).

\subsection{UAV-borne laser scanning}

UAV-borne laser scanning (ULS) is a dynamic, active remote sensing technique, with a scanner mounted on top of a UAV. During a measurement campaign, the trajectory is continuously recorded with the help of an inertial measurement unit (IMU) and a differential global navigation satellite system (DGNSS). During the flight, emitted laser pulses are deflected by a rotating mirror orthogonal to the movement direction. Depending on the aim of the ULS campaign, the footprint size and the point density can be adapted during flight planning. ULS combines the advantages of ALS (area-wide coverage with view from above) and TLS (flexibility and high resolution and accuracy). However, the spatial extent of ULS campaigns is limited to a few square kilometers. Furthermore, legal restrictions regarding the maximum flight altitude of $150 \mathrm{~m}$, the maximum distance to the pilot of $500 \mathrm{~m}$ and a continuous visual line of sight (VLOS) must be considered.

For the ULS campaign at the Vögelsberg landslide on 3rd August 2018 a RIEGL RiCOPTER equipped with a VUX-1LR laser scanner, an Applanix AP20 IMU/DGNSS unit and two Sony Al-

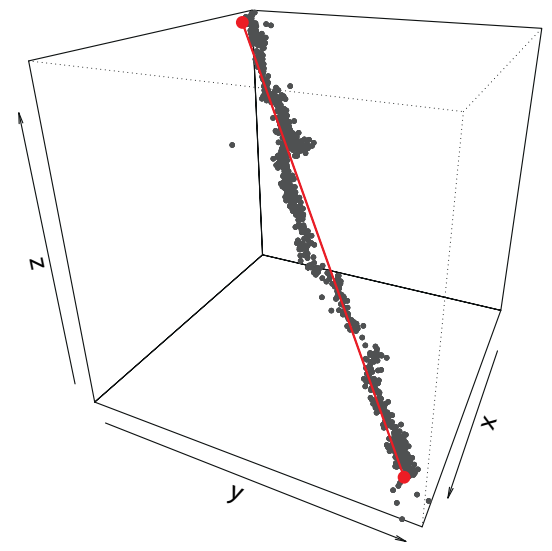

Figure 3. Fitted 3D displacement vector (red line with start and end points, first principle component) based on daily ATTS measurements. 
pha 6000 digital cameras was used. The octocopter has a maximum payload of $16 \mathrm{~kg}$ (sensors and batteries) and can fly for 30 minutes with one set of batteries. The VUX-1LR scanner has a maximum range of $1.500 \mathrm{~m}$ and a nominal measurement accuracy of $15 \mathrm{~mm}$. Laser pulses are emitted in the near infrared with a pulse repetition rate (PRR) of up to $820 \mathrm{kHz}$ and a beam divergence of $0.5 \mathrm{mrad}$.

The area of interest was covered by five individual flights (Fig. 2) with approximately ten minutes of scanning time each. The flights include 18 longitudinal strips following the direction of the valley and two cross strips. Before and after each flight five minutes of static and two minutes of dynamic alignment were carried out. The flights were planned with the UgCS Software (UGCS, 2019). Within the area of interest the acquired 3D point cloud should have minimum point density of 500 points per square meter and a positional accuracy of $4.0 \mathrm{~cm}$. For the scanning a pulse repitition rate of $820 \mathrm{kHz}$ and an angular step width of $0.0587^{\circ}$ was chosen. The flight altitude was held between 50 to $60 \mathrm{~m}$ at a speed of about $8 \mathrm{~m} / \mathrm{s}$.

\subsection{Data processing}

Figure 4 shows the main data processing steps carried out in the present study. All processing steps for the available ALS point cloud were conducted with the software SAGA (Conrad et al., 2015) with the LIS Pro 3D (Laserdata, 2019; Rieg et al., 2014) extension. For an efficient data processing a data management including a virtual point cloud based on the Extensible Markup Language (XML) was set up. A virtual point cloud is a metadata file listing point cloud tiles and their extent, allowing to access them seamlessly. In case of the Vögelsberg dataset tiles of $500 \times 500 \mathrm{~m}$ and an additional overlap of $20 \mathrm{~m}$ were generated. The overlap was necessary for the subsequent ground classification following the progressive TIN densification approach (Axelsson, 2000). From the points classified above-ground planar areas were segmented with a plane growing algorithm implemented in SAGA LIS Pro 3D (Laserdata, 2019). Seed points for the plane growing were selected using a threshold for the locally computed planarity feature. This feature is based on eigenvectors computed within a defined neighbourhood (in the present study a radius of $1.0 \mathrm{~m}$ was used) and can be used as a measure of surface roughness. The planarity $p_{i}$ of point $i$ is defined as

$$
p_{i}=\frac{e_{i 1}+e_{i 2}}{e_{i 1}}
$$

where $e_{i 1}$ is the eigenvalue of the longest and $e_{i 2}$ is the eigenvalue of the second longest eigenvector within the considered neighbourhood. The planarity ranges from 0 to 1 and is particularly high on planar areas like roofs or walls. However, the walls, which are well represented in the ULS data were omitted because they are not present in the ALS data. The resulting roof segments were then aggregated using a density-based spatial clustering algorithm (Ester et al., 1996).

The acquired ULS data require several pre-processing steps. First, the trajectory recorded by the IMU/GNSS unit was differentially corrected using the software Applanix POSPac (Applanix, 2019) and reference data of the closest permanent DGNNS reference station of the Austrian Positioning Service (APOS) situated at Innsbruck Airport. Thereby, the lever arm $(x=0.170 \mathrm{~m}, y=-0.020 \mathrm{~m}, z=-0.267 \mathrm{~m})$ from the GNSS antenna on top of the octocopter to the IMU centre was

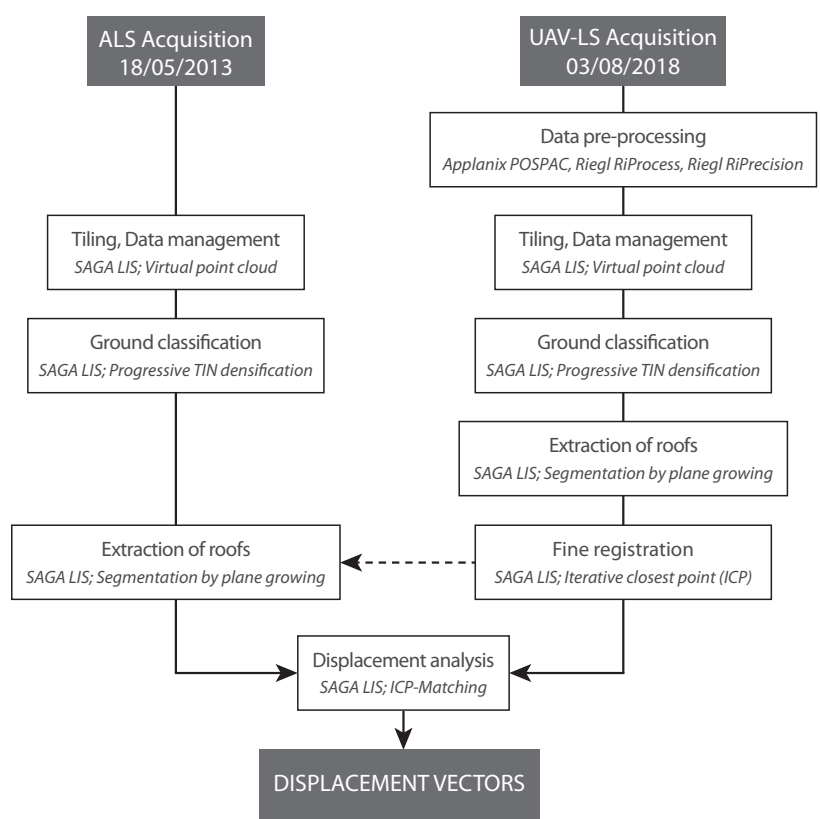

Figure 4. Workflow showing the main data processing steps.

considered. After trajectory correction, the single strip point clouds were generated by offline waveform processing and georeferencing in the Riegl Software Packages RiAnalyse and RiWorld, run from the software RiProcess (Riegl LMS, 2019). Finally, the input trajectories and point clouds were improved by a strip-adjustment implemented in the software package RiPrecision (Riegl LMS, 2019). In total, more than 900 million points were acquired.

All further processing steps were conducted with the software SAGA LIS Pro 3D (Laserdata, 2019) which are generally similar to the processing workflow of the ALS data. However, in case of the ULS data smaller tiles of $100 \times 100 \mathrm{~m}$ with an overlap of $10 \mathrm{~m}$ were generated because of the distinctly higher point density. The ground classification and segmentation of roofs were conducted with the same tools as described for the ALS data. In contrast to the ALS data, the ULS point cloud also includes vertical walls of buildings. Segments representing roofs were separated from vertical walls by defining a threshold for the angle of the eigenvector corresponding to the smallest eigenvalue, assuming that roofs are inclined between 0 to 60 degrees. Based on the roofs located on stable grounds a fine registration of the ULS and the ALS data was applied using the iterative closest point (ICP) algorithm (Besl and McKay, 1992).

The roofs extracted from the multi-temporal laser scanning data were then used to derive the respective displacement vectors with the help of a recently introduced algorithm (ICP-Matching) based on the ICP algorithm (Besl and McKay, 1992). The ICPMatching algorithm was designed to quantify changes of two 3D point clouds, which occurred between their acquisitions (Fig. 5). It was introduced and applied in a previous study to assess displacements of a deep-seated landslide in an unbuilt area (Pfeiffer et al., 2018). The ICP-Matching algorithm iteratively matches the points of one point cloud (slave) to the points of the second point cloud (master) and builds point to plane correspondences (Chen and Medioni, 1992). These connections of points in the master dataset to planes of the slave dataset are exported as $3 \mathrm{D}$ vectors, which represent changes between the two point clouds. In the present study, the ICP-Matching algorithm was applied object- 
Table 1. Meta data for the used ALS and ULS data including the number of points and mean point density for the considered total area covering the active landslide and its surroundings and the extracted roofs.

\begin{tabular}{|c|c|c|c|c|c|c|c|}
\hline \multirow[b]{2}{*}{ Epoch } & \multirow[b]{2}{*}{ Acquisition date } & \multirow[b]{2}{*}{ Platform } & \multirow{2}{*}{ Sensor } & \multicolumn{2}{|c|}{ Number of points } & \multicolumn{2}{|c|}{ Mean point density $\left[\mathrm{pts} / \mathrm{m}^{2}\right]$} \\
\hline & & & & Total area & Extracted roofs & Total area & Extracted roofs \\
\hline 1 & $2013 / 05 / 18$ & Eurocopter AS350 & RIEGL LMS-Q680i & 49.145 .177 & 322.255 & 33 & 24 \\
\hline 2 & $2018 / 08 / 03$ & RIEGL RiCOPTER & RIEGL VUX-1LR & 272.337 .014 & 1.804 .037 & 265 & 133 \\
\hline
\end{tabular}

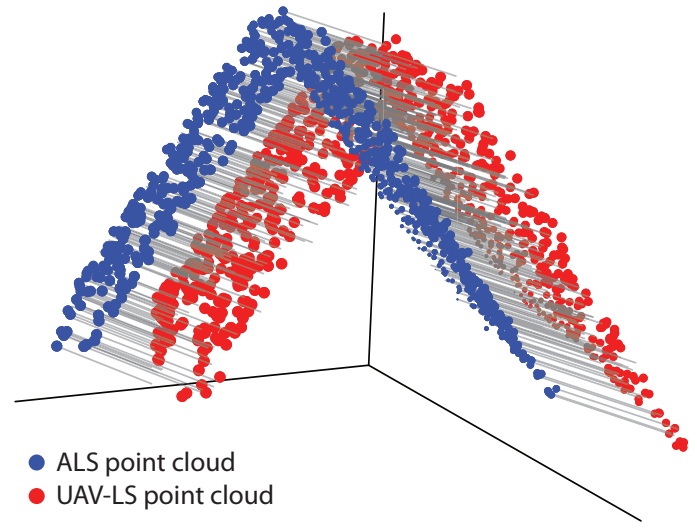

Figure 5. Principle of the segment-based ICP-Matching algorithm.

based for each extracted roof to derive their individual changes. Before applying the ICP-Matching tool, all roofs were checked for structural modifications (e.g. added solar panels) between the two time steps based on available orthophoto series. Such modifications could bias the resulting displacement vectors. However, no modifications of roofs or on top of roofs were observed in the study area. With the ICP-Matching algorithm numerous displacement vectors were derived for each considered roof. For the comparison with the ATTS measurements mean annual vectors were computed for each roof.

\section{RESULTS}

In total, 200.348 vectors were derived for 35 roofs extracted from the multi-temporal laser scanning data. The number of vectors for the individual roofs range between 1.072 and 17.683 vectors. As a measure of accuracy, distances of the points to planes locally fitted to the ULS data were computed for both laser scanning datasets considering the extracted roofs. In case of the ULS data the standard deviation of these distances amounts to $1.5 \mathrm{~cm}$. The standard deviation of the distances of the ALS points to the same planes is $3.2 \mathrm{~cm}$. Therefore, displacements below about $5 \mathrm{~cm}$ (1 standard deviation) could not be detected over the covered period of more than 5 years beyond doubt, corresponding to about $1 \mathrm{~cm} \mathrm{a}^{-1}$. This is also supported by the computed root mean squared error (RMSE) of the ICP-matched roofs, which is $5.2 \mathrm{~cm}$.

Figure 6 shows the displacement vectors derived from the ATTS measurements and the mean displacement vectors based on the ICP-Matching algorithm using the multi-temporal laser scanning data. Outside the active area vectors derived from the ATTS point in north-western direction with minor horizontal and vertical magnitudes. Respective vectors derived from the laser scanning data outside the active area are pointing in random directions, also with minor horizontal and vertical magnitudes. Within the active area the resulting vectors from both monitoring techniques are directed downslope with comparable magnitudes. The vertical vectors indicate a lowering of the affected roofs. The mean total displacement within the unstable area derived from the ATTS measurements is $6.4 \mathrm{~cm}$, corresponding to $2.9 \mathrm{~cm} \mathrm{a}^{-1}$ for the observation period of 2.2 years. The mean total displacement within the unstable area derived from the multi-temporal laser scanning data is $13.2 \mathrm{~cm}$, resulting in $2.4 \mathrm{~cm} \mathrm{a}^{-1}$ for the covered period of 5.4 years.

The direction and magnitude of the derived 3D displacement vectors are compared in the wind rose plots in Fig. 7. In case of the results of the laser scanning data vectors with a minor magnitude below $15 \mathrm{~mm} / \mathrm{a}$ point in arbitrary directions (Fig. 7a). These short vectors are within the data uncertainty and the objects they are representing can be considered stable. As shown in the map in Fig. 6 these vectors are mainly located outside the active area. Displacement vectors between 15 and $45 \mathrm{~mm} / \mathrm{a}$ point in the north to north-eastern direction. Displacement vectors derived from the ATTS measurements, which are below $15 \mathrm{~mm} / \mathrm{a}$ are pointing in the eastern to north-eastern direction. Also vectors of 15 to $30 \mathrm{~mm} / \mathrm{a}$ length are pointing in this direction. Longer vectors of 15 to $45 \mathrm{~mm} / \mathrm{a}$ are pointing in the north to north-eastern direction, which is in general agreement with the results of the analysis of the laser scanning data.

Figure 8 shows the 3D lengths (a,b) and the vertical component $(\mathrm{c}, \mathrm{d})$ of the derived displacement vectors, classified according to their location in the active (unstable) area or in the surrounding stable area. The longest vectors are located in the active area in case of the results based on the laser scanning data (Fig. 8a) and the ATTS measurements (Fig. 8b). At least landslide displacements above $30 \mathrm{~mm} / \mathrm{a}$ are clearly detectable and discernible from the stable grounds. Mean annual displacements derived from the ATTS measurements are generally higher than those based on the laser scanning data. However, not all measurement points are covered by both monitoring techniques, prohibiting a quantitative comparison.

Also the highest vertical changes are located in the active, unstable area in case of the results based on the laser scanning data (Fig. 8c) and the ATTS measurements (Fig. 8d). The vertical component within the active area is lower than $-5 \mathrm{~mm} / \mathrm{a}$ for the laser scanning data (Fig. 8c) and can be discerned from the vertical changes in the stable part of the slope. There, vertical changes range from slightly negative to slightly positive. The vertical components of the vectors derived from the ATTS measurements indicate a greater downward movement of up to $-24 \mathrm{~mm} / \mathrm{a}$. Also the measurement point on stable grounds show a minor sinking.

Additional information, which can be derived from the multitemporal laser scanning data is the area-wide 3D deformation of the extracted roofs (Fig. 9). Gradual changes of vector lengths within single roofs indicate rotational movements along the gradient. Some houses show a tilting movement in the direction of the landslide movement. Particularly the buildings located on the lower part of the slope are affected by differential movements. 


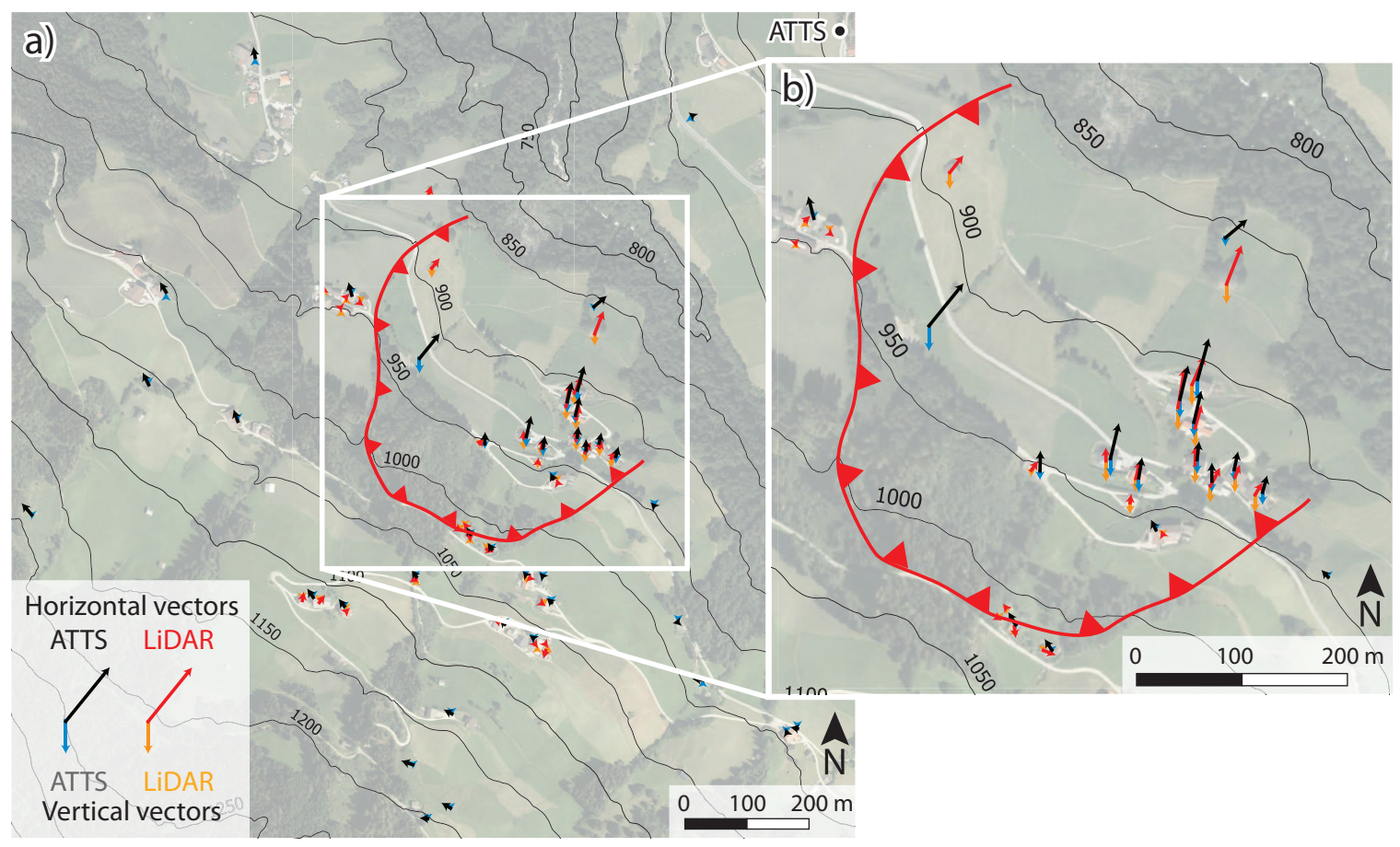

Figure 6. Annual horizontal and vertical displacement vectors derived from the laser scanning data (horizontal in red, vertical in orange) and the ATTS (horizontal in black, vertical in blue) for the study area (a) and a detail view of the active area (b). For visualization purposes the length of the vectors is scaled up by the factor 1000 .
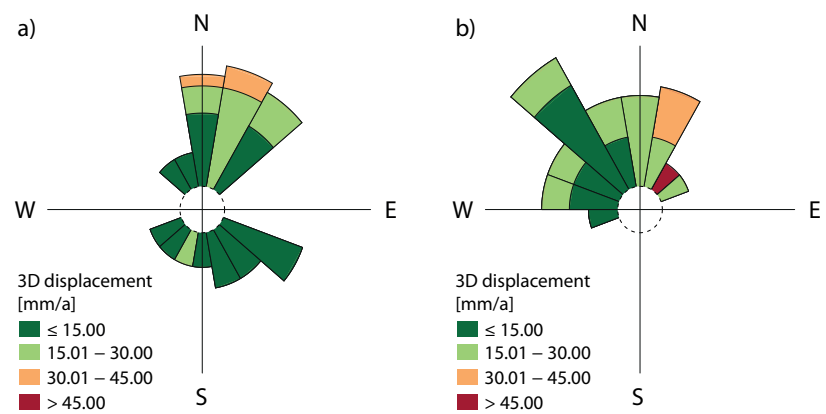

Figure 7. Classified direction and magnitude of the annual displacement vectors derived from the laser scanning data (a) and the ATTS (b).

\section{DISCUSSION}

The comparison of the results of the two monitoring techniques is limited by the fact that different periods are covered by the data. A quantitative comparison is therefore based on the assumption that the landslide movement is continuous and constant. In addition, not all measurement points were covered by both techniques. Furthermore, on the buildings, the retroreflective prisms were mainly mounted to the walls, while the analysis of the laser scanning data focused on the extracted roofs. This raises the question what kind of displacement is derived from both techniques. Landslide displacements can be measured at the sliding surface, on the landslide surface and with the help of aboveground objects travelling on top of the landslide. Depending on the type of landslide and type of objects the respective displacements can deviate. Particularly buildings may exhibit internal deformation biasing the estimate of the actual landslide-induced movement. Such internal deformation is depending on the used

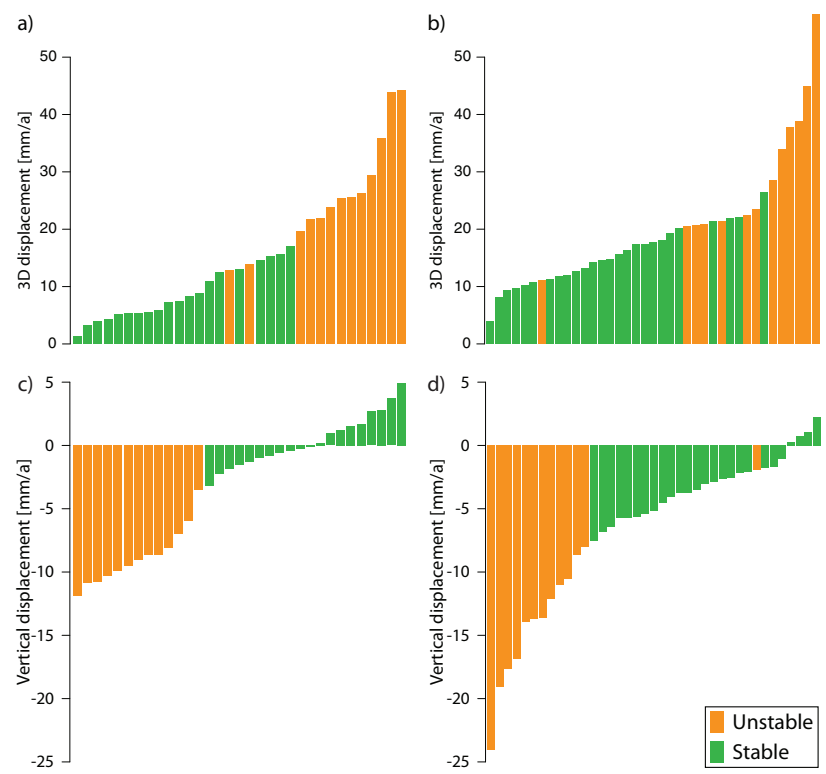

Figure 8. 3D length $(a, b)$ and vertical component $(c, d)$ of the displacement vectors derived from the laser scanning data $(a, c)$ and the ATTS measurements $(b, d)$.

materials and may even show a seasonal trend.

The mean displacement vectors computed for each roof extracted from the multi-temporal laser scanning data are based on highly redundant data with 1.072 to 17.683 individual vectors, depending on the (covered) area of the roof and the local point density. It could be argued that the accuracy and robustness of the derived mean displacement vectors correlates with redundancy. However, the computed RMSE of the matched roofs did not show a trend 


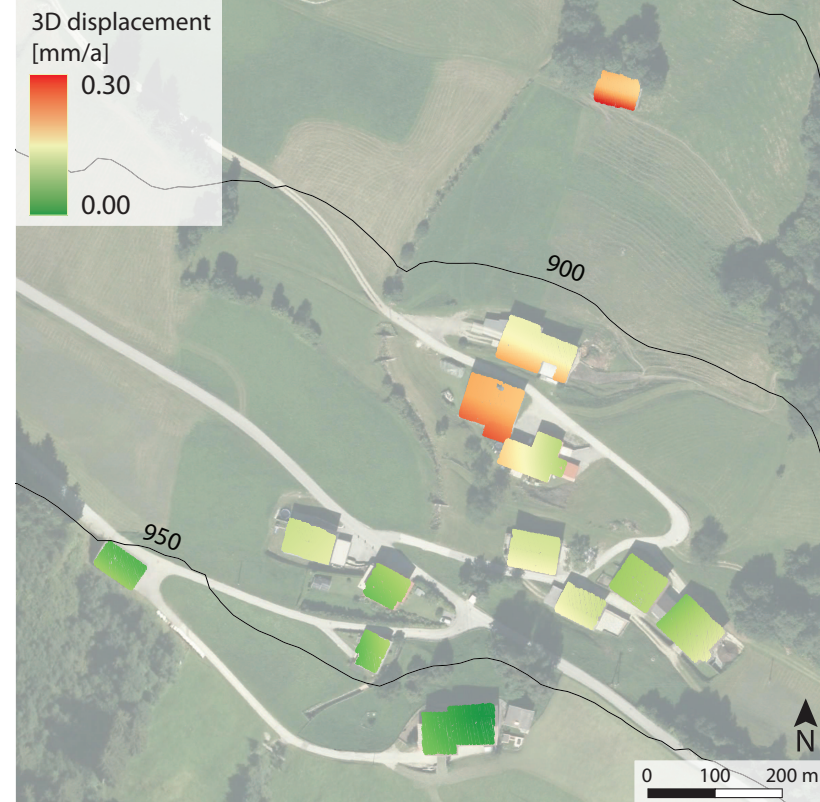

Figure 9. Derived 3D displacement vectors derived from the laser scanning data showing deformation of extracted roofs in the area affected by the active landslide.

when compared to the respective number of vectors. This may be related to the generally large number of individual vectors.

The apparent discrepancy of the vectors' directions in the unstable area could be explained by the geometry of the extracted roofs. For deriving 3D displacement vectors from the roofs, their geometry should include planes pointing in at least three directions. This is not the case for saddle roofs which make up half of the roofs in the active area. To account for these cases, the walls which are represented in the ULS data should be considered for the displacement analysis. However, since walls are not present in the ALS data, point clouds derived from another ULS campaign and from terrestrial laser scanning will be included in future studies.

\section{CONCLUSIONS}

The presented comparison of the results is limited by the facts that (i) additional targets are considered by the ATTS (e.g. on installed poles), (ii) the considered period of time differs, (iii) constant displacement rates are assumed for the comparison and (iv) internal deformations of the buildings are neglected. However, the results of the study showed that in the case of the Vögelsberg site landslide-induced displacements can be efficiently assessed based on roofs represented in multi-temporal laser scanning data using the presented ICP-Matching algorithm. The direction and magnitude of the derived displacement $3 \mathrm{D}$ vectors generally agree with measurements of an ATTS. However, buildings can exhibit internal deformation, which will add to the landslideinduced movement.

To account for the internal deformation of objects the vertical walls should be considered in the analysis. Multi-temporal ULS data or periodical terrestrial laser scans may be suitable for assessing both, landslide displacements and the deformation of monitored objects. Furthermore, the displacement monitoring could be extended to other above-ground objects such as poles, fences or trees in future.

\section{ACKNOWLEDGEMENTS}

The present study was conducted within the OPERANDUM project. This project has received funding from the European Union's Horizon 2020 research and innovation programme under grant agreement No 776848. We thank the Austrian Service for Torrent and Avalanche Control (WLV), the Austrian Research Centre for Forests (BFW) and the federal state of Tyrol, division of geoinformation for their cooperation. We also thank the municipal administrator and the land owners for their support. The Unmanned aerial vehicle Laser Scanning data has been acquired by the 4D LiDAR mountAin Monitoring laB (https://4dlamb.mountainresearch.at/) funded by the Federal Ministry of Education, Science and Research, Republic of Austria.

\section{References}

Applanix, 2019. www.applanix.com. Last accessed: 12 January 2019.

Axelsson, P., 2000. DEM generation from laser scanner data using adaptive TIN models. International Archives of Photogrammetry and Remote Sensing 33(4), pp. 110-117.

Besl, P. J. and McKay, N. D., 1992. A method for registration of 3-D shapes. IEEE Transactions on pattern analysis and machine intelligence 14(2), pp. 239-256.

Břežný, M. and Pánek, T., 2017. Deep-seated landslides affecting monoclinal flysch morphostructure: Evaluation of LiDARderived topography of the highest range of the Czech Carpathians. Geomorphology 285, pp. 44-57.

Chen, Y. and Medioni, G., 1992. Object modelling by registration of multiple range images. Image and Vision Computing 10(3), pp. $145-155$.

Conrad, O., Bechtel, B., Bock, M., Dietrich, H., Fischer, E., Gerlitz, L., Wehberg, J., Wichmann, V. and Böhner, J., 2015. System for Automated Geoscientific Analyses (SAGA) v. 2.1.4. Geoscientific Model Development 8(7), pp. 1991-2007.

Cruden, D. M. and Varnes, D. J., 1996. Landslide types and processes. In: A. Turner and R. Schuster (eds), Landslides: Investigation and Mitigation, Transportation Research Board Special Report 247. US National Research Council, Washington, DC, p. 36-75.

Eeckhaut, M. V. D., Poesen, J., Verstraeten, G., Vanacker, V., Nyssen, J., Moeyersons, J., Beek, L. P. H. v. and Vandekerckhove, L., 2007. Use of LIDAR-derived images for mapping old landslides under forest. Earth Surface Processes and Landforms 32(5), pp. 754-769.

Ester, M., Kriegel, H.-P., Sander, J. and Xu, X., 1996. A density-based algorithm for discovering clusters in large spatial databases with noise. In: Proceedings of 2nd International Conference on Knowledge Discovery and Data Mining (KDD 96), AAAI Press, Portland, Oregon, pp. 226-231.

Gauthier, D., Anderson, S. A., Fritz, H. M. and Giachetti, T., 2018. Karrat Fjord (Greenland) tsunamigenic landslide of 17 June 2017: initial 3D observations. Landslides 15(2), pp. 327 332.

Jaboyedoff, M., Oppikofer, T., Abellan, A., Derron, M.-H., Loye, A., Metzger, R. and Pedrazzini, A., 2012. Use of LIDAR in landslide investigations: a review. Natural Hazards 61(1), pp. 5-28. 
Laserdata, 2019. LIS Pro 3D software package, www.laserdata.at/software.html. Last accessed: 12 January 2019.

Lin, Y., Hyyppä, J. and Jaakkola, A., 2011. Mini-UAV-Borne LIDAR for Fine-Scale Mapping. IEEE Geoscience and Remote Sensing Letters 8(3), pp. 426-430.

Mandlburger, G., Wenzel, K., Spitzer, A., Haala, N., Glira, P. and Pfeifer, N., 2017. IMPROVED TOPOGRAPHIC MODELS VIA CONCURRENT AIRBORNE LIDAR AND DENSE IMAGE MATCHING. ISPRS Ann. Photogramm. Remote Sens. Spatial Inf. Sci. IV-2/W4, pp. 259-266.

Mayr, A., Rutzinger, M., Bremer, M., Elberink, S. O., Stumpf, F. and Geitner, C., 2017. Object-based classification of terrestrial laser scanning point clouds for landslide monitoring. Photogrammetric Record 32(160, SI), pp. 377-397.

Monserrat, O. and Crosetto, M., 2008. Deformation measurement using terrestrial laser scanning data and least squares 3D surface matching. ISPRS Journal of Photogrammetry and Remote Sensing 63(1), pp. 142-154.

Pánek, T. and Klimeš, J., 2016. Temporal behavior of deep-seated gravitational slope deformations: A review. Earth-Science Reviews 156 , pp. 14-38.

Pfeiffer, J., Zieher, T., Bremer, M., Wichmann, V. and Rutzinger, M., 2018. Derivation of Three-Dimensional Displacement Vectors from Multi-Temporal Long-Range Terrestrial Laser Scanning at the Reissenschuh Landslide (Tyrol, Austria). Remote Sensing.

Rieg, L., Wichmann, V., Rutzinger, M., Sailer, R., Geist, T. and Stötter, J., 2014. Data infrastructure for multitemporal airborne LiDAR point cloud analysis - Examples from physical geography in high mountain environments. Computers, Environment and Urban Systems 45, pp. 137-146.
Riegl LMS, 2019. www.riegl.com. Last accessed: 12 January 2019.

Riquelme, A. J., Abelian, A. and Tomas, R., 2015. Discontinuity spacing analysis in rock masses using 3D point clouds. Engineering Geology 195, pp. 185-195.

Telling, J., Lyda, A., Hartzell, P. and Glennie, C., 2017. Review of Earth science research using terrestrial laser scanning. Earth-Science Reviews 169, pp. 35-68.

UGCS, 2019. www.ugcs.com. Last accessed: 12 January 2019.

Wallace, L., Lucieer, A., Watson, C. and Turner, D., 2012. Development of a UAV-LiDAR System with Application to Forest Inventory. Remote Sensing 4(6), pp. 1519-1543.

Wieser, M., Hollaus, M., Mandlburger, G., Glira, P. and Pfeifer, N., 2016. ULS LiDAR SUPPORTED ANALYSES OF LASER BEAM PENETRATION FROM DIFFERENT ALS SYSTEMS INTO VEGETATION. ISPRS Ann. Photogramm. Remote Sens. Spatial Inf. Sci. III-3, pp. 233-239.

Wujanz, D., Avian, M., Krueger, D. and Neitzel, F., 2018. Identification of stable areas in unreferenced laser scans for automated geomorphometric monitoring. Earth Surface Dynamics 6(2), pp. 303-317.

Zieher, T., Toschi, I., Remondino, F., Rutzinger, M., Kofler, C., Mejia-Aguillar, A. and Schlögel, R., 2018. SENSORAND SCENE-GUIDED INTEGRATION OF TLS AND PHOTOGRAMMETRIC POINT CLOUDS FOR LANDSLIDE MONITORING. Int. Arch. Photogramm. Remote Sens. Spatial Inf. Sci. XLII-2, pp. 1243-1250. 